\title{
Trans-arterial embolization of malignant tumor-related gastrointestinal bleeding: technical and clinical efficacy
}

\author{
Ali H. Elmokadem ${ }^{1 *}$ (D), Hassan Abdelsalam² ${ }^{2}$ Ahmed El-Morsy ${ }^{1}$ and Ahmed Elsabbagh ${ }^{3}$
}

\begin{abstract}
Background: Gastrointestinal (Gl) tract bleeding is a major cause of mortality among patients with GI malignancies. We aimed to assess the technical and clinical efficacy of trans-arterial embolization (TAE) as a symptomatic treatment of tumor-related Gl bleeding. This study was conducted for patients with Gl bleeding secondary to histopathologically proven different GI malignancies. Fourteen patients underwent trans-arterial embolization. Patients were followed up clinically for any complications or episodes of recurrent bleeding.

Results: Fourteen patients were included (9 males and 5 females) with mean age 55.5 years (range 42-69 years). All procedures were technically successful with post-procedural hemorrhage control and no immediate complication. The 30-day post-procedural clinical success rate was $78.4 \%$. Three repeated clinically successful TAE sessions were done for recurrent bleeding. The median post-procedural follow-up duration was 241 days. The 30-day mortality rate was $7.1 \%$, while the overall mortality rate was $35.7 \%$.
\end{abstract}

Conclusion: Trans-arterial embolization of tumor-related Gl bleeding controlled hemorrhage with acceptable clinical success rate and without complication in this small group of patients.

Keywords: Endovascular, Embolization, GIT, Bleeding, Tumor

\section{Background}

Tumor-related gastrointestinal (GI) tract bleeding accounts for less than $5 \%$ of upper GI hemorrhages [1] and $3-11 \%$ of lower GI hemorrhages [2]. Upper gastrointestinal (GI) bleeding is defined as occurring anywhere from the esophagus to the duodenojejunal flexure at the attachment of the ligament of Treitz, and beyond this level is considered lower GI bleeding [3]. Different tumors include primary tumors such as adenocarcinoma, lymphoma, and GI stromal tumors (GISTs) as well as metastatic intestinal tumors have been reported as sources of acute and chronic GI hemorrhage.

Nonvariceal upper GI bleeding is often caused by gastric or duodenal ulcers, gastritis, or duodenitis and less commonly is secondary to vascular malformations, esophageal tears, and tumors. The urgent therapy of acute, massive upper GI nonvariceal bleeding is based

\footnotetext{
*Correspondence: mokadem83@yahoo.com

${ }^{1}$ Diagnostic \& Interventional Radiology Department, Mansoura University,

Elgomhoria St, Mansoura, Dakahlia 35516, Egypt

Full list of author information is available at the end of the article
}

on identifying and occluding the bleeding artery. Typically, endoscopy is the first-line in diagnosing and treating acute GI bleeding, yet its benefit for the management of bleeding GI tumors is questionable due to high recurrence and complication rates [4-7]. Endoscopic management rarely eliminates bleeding or decrease overall mortality. Radiotherapy has a vital role in the management of malignant GI neoplasms, both in primary and palliative therapies. Nevertheless, data reporting radiotherapy for the treatment of bleeding GI malignant tumors are limited. Radiotherapy is considered ineffective for acute hemodynamically significant hemorrhage, but it may provide some benefit in the cases of chronic blood loss [7]. Many articles reported high morbidity and mortality rates associated with surgical resection of advanced GI tumors [7-9]. Even with limited effectiveness of surgical and endoscopic management of neoplastic GI hemorrhage, there is a lack of literature regarding trans-arterial embolization for tumorrelated GI hemorrhage. We report our initial experience to assess the feasibility and technical and clinical 
effectiveness of endovascular management in patients with tumor-related GI bleeding.

\section{Methods}

The Institutional Review Board approved this retrospective study. Written consent was obtained from all patients before angiography and embolization after clarification of the possible hazards of the technique. The study population consisted of 14 patients $(9$ males and 5 females) with a mean age 55.5 years (range 42-69 years) who were referred for interventional radiology for management in two centers. The patients had signs of GI hemorrhage, such as melena or hematemesis with histopathologic proof of GI malignancy. Data were extracted from medical records. Radiologic, endoscopic, and pathologic reports were reviewed to identify the tumors' type and location. Eight patients had known gastric adenocarcinoma, three patients with gastric lymphoma, two patients with gastrointestinal stromal tumor (GIST), and one patient with post-operative recurrent colonic adenocarcinoma which invaded the duodenum.

Endoscopic evaluation was done for bleeding secondary to gastric tumors. Morphology on endoscopy was classified using the Borrmann classification system (Type I, polypoid fungating mass; Type II, ulcerative mass with elevated distinct borders; Type III, ulcerative mass with indistinct borders; Type IV, diffuse infiltration, indistinct borders) [10]. The Forrest classification was applied for describing the bleeding foci (stage I represents active hemorrhage: Forrest I a (spurting hemorrhage) and Forrest I b (oozing hemorrhage); stage II shows signs of recent hemorrhage: Forrest II a (non-bleeding Visible vessel), Forrest II b (adherent clot), and Forrest II c (flat pigmented hematin on ulcer base); stage III describes lesions without active bleeding or fibrin-covered clean ulcer base) [11]. Active bleeding or signs of recent bleeding were noted on endoscopy in all cases; an oozing type of bleeding (Forrest I b, $n=7$ ) was more frequently observed than spurting bleeding (Forrest I a, $n=2$ ). Two patients showed signs of recent bleeding with adherent clots (Forrest II b). The decision of whether or not to attempt endoscopic hemostasis was made by the gastroenterologist. In 9 of the 11 patients with active bleeding, endoscopic hemostasis was performed. In the remaining 2 patients with adherent clots, hemostatic procedures were not undertaken owing to technical difficulties. All patients who had endoscopic treatment showed persistent bleeding after the procedures, and hence they were referred to endovascular management.

All patients received blood transfusion and were clinically stable prior to the endovascular procedures. The endovascular procedures were done on (Philips, AlluraClarity system, the Netherlands). All procedures were done by experienced intervention radiologists ( 8 and 15 years of experience) under local anesthesia through trans-femoral route using a 6-Fr sheath and 5-Fr catheters (Simmons II or Cobra, Cook Medical, Bjaeverskov, Denmark). Pre-embolization angiograms were obtained to evaluate the tumor blush and neovascularity as well as contrast extravasation.

In all patients with bleeding gastric tumors (illustrative case; Fig. 1), the celiac artery was catheterized with a 5Fr Cobra catheter with superselection of the left gastric or right gastroepiploic arteries using a microcatheter (Progreat 2.7 Fr, Terumo Europe, Leuven, Belgium or Renegade; Boston Scientific, Natick, Massachusetts). Particle embolization was done in all cases using polyvinylalcohol (PVA) particles (Contour SE; Boston Scientific) between 355 and $500 \mu \mathrm{m}$ in size or gelatin sponge particles (Upjohn company, Kalamazoo, MI, USA). The choice of embolic material was dictated by the operator's

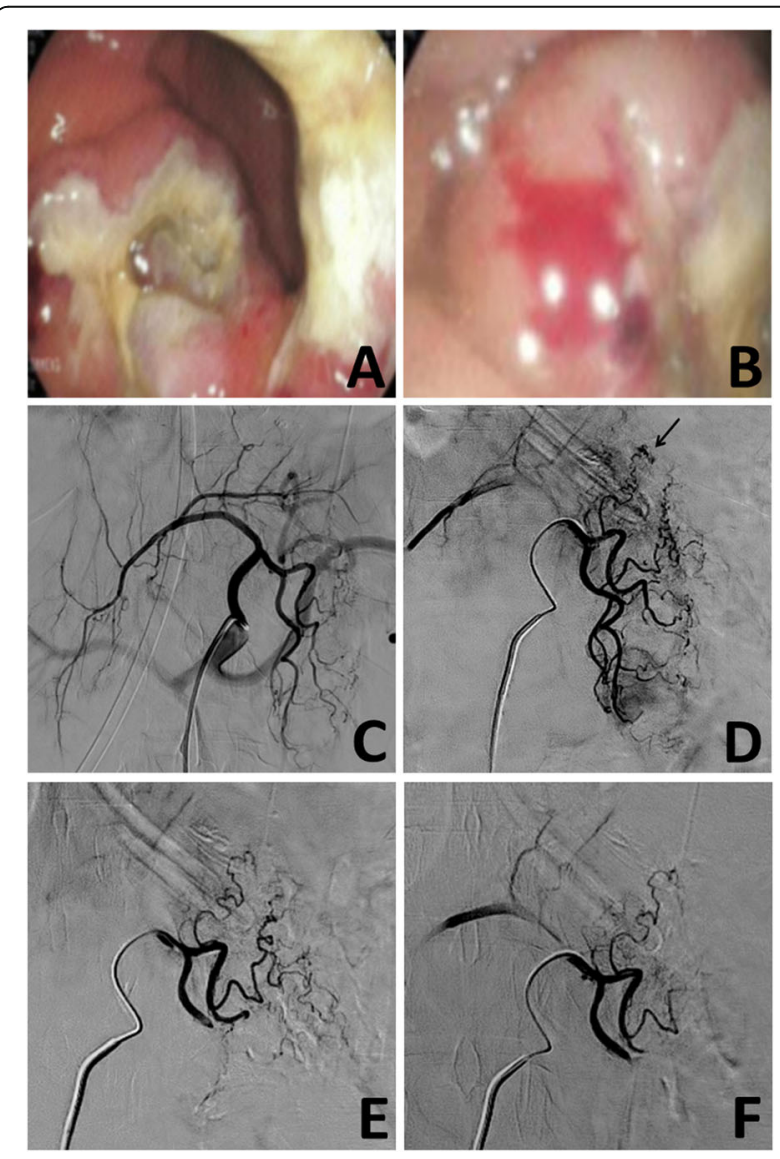

Fig. 1 a, b Endoscopic examination revealed large irregular gastric mass with bleeding on its surface. $\mathbf{c}$ Left gastric angiogram shows the tumor neovascularity and blush on the left side. $\mathbf{d}$ Angiogram obtained after selective micro-catheterization of the feeding artery shows the dense tumor blush, distal luminal irregularity, and extravasation (black arrow). e, f Consecutive post-embolization angiograms demonstrate stasis with absent tumor blush 
preference. Particles were infused until stasis was achieved.

Small bowel bleeding occurred in three cases including two cases of intestinal GIST; the CT scan for one patient showed a massive GIST tumor supplied predominantly from the gastroduodenal artery (GDA) with multiple superior mesenteric artery (SMA) branches (Fig. 2), while the other one had exclusive supply from the GDA. In both cases, the feeding arteries were catheterized with Progreat microcatheter and embolized with PVA particles $(355-500 \mu \mathrm{m})$ until stasis was achieved. The third patient had local recurrence from transverse colon adenocarcinoma which invaded the duodenum (Fig. 3) and supplied from the GDA. The feeding artery was catheterized with similar fashion as previously described and embolized with gelatin sponge particles.

The particles were infused slowly under fluoroscopy until stasis of flow in the desired artery. Completion angiograms were done to demonstrate the absence of residual tumor blush. Post-angiography clinical notes were reviewed to ascertain the occurrence of complications and episodes of recurrent bleeding. Technical success was defined as acquiring stasis in the targeted arteries. Clinical success was defined as the resolution of signs and symptoms of GI bleeding within $24 \mathrm{~h}$ after the procedure, with continued absence for 30 days following embolization. If the patient received blood/red blood cell transfusions, underwent invasive procedures to address continued GI bleeding, or admitted for recurrent GI bleeding within 30 days, the procedure was deemed a clinical failure.

Descriptive statistics were used to characterize the demographic features of the study population, and the continuous variables were expressed as mean \pm standard deviation. Categorical variables were expressed as the case number (percentage). The Kaplan-Meier method was used to assess the cumulative survival period from the time of the initial management.

\section{Results}

Fourteen cases were included in this study to manage GI bleeding secondary to histopathologically proven GI malignancy visualized by either endoscopy or CT scan. In 11 patients, the bleeding source was gastric tumors (adenocarcinoma and lymphoma), and 3 patients bled from small bowel tumors (GIST and adenocarcinoma). All cases were stabilized and had undergone packed red blood cell or whole blood transfusions prior to the endovascular procedure. Nine patients with gastric tumors had one failed trial of endoscopic management of bleeding prior to referral for transcatheter embolization (TAE). Angiographic identification of the tumor by blush was noted in 12 cases, neovascularity in 9 cases and active extravasation was seen in 2 cases. The baseline demographics, presentations, tumor type, location, angiographic features, and management were summarized in Table 1.

Technical success was reported in all procedures. Clinical success was achieved in 11 procedures (78.4\%). The bleeding recurred in three patients at 15, 20, and 26 days after embolization procedures. The recurrent bleeding was managed conservatively in two patients and repeat embolization procedure was done in one case. Two repeated clinically successful TAE sessions were done to control recurrent GI bleeding at 65 and 135 days after initial procedures in cases of gastric lymphoma. All repeat procedures were done using PVA particles and they were clinically successful. All patients developed

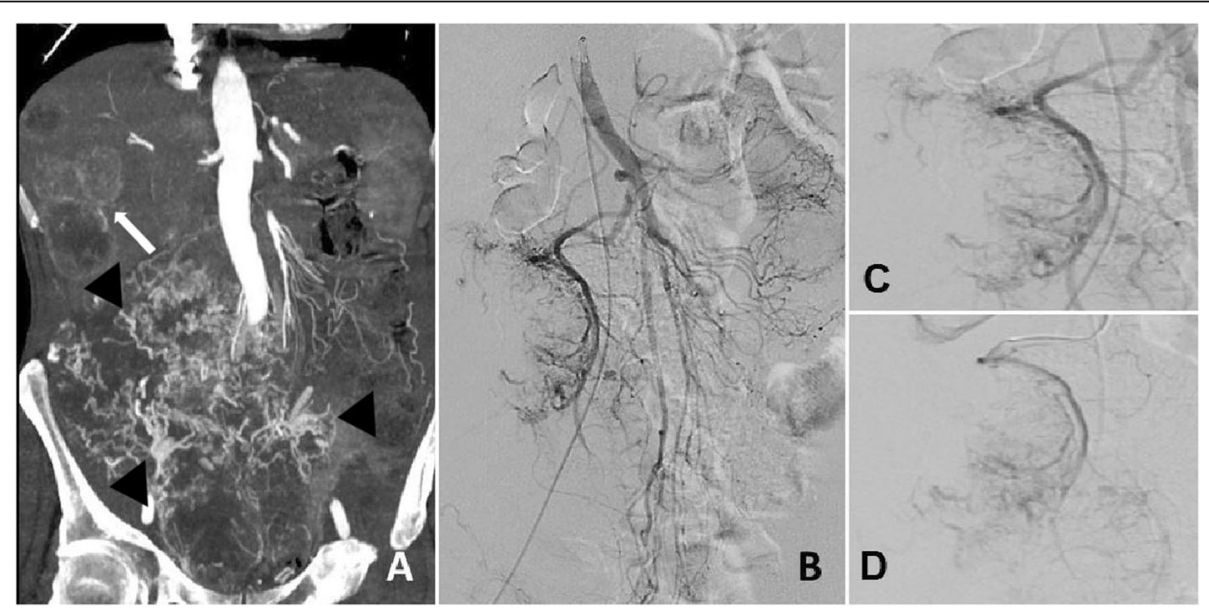

Fig. 2 a Coronal maximum intensity projection (MIP) reformatted CT image for a patient with massive small bowel GIST shows the extensive vascularity of the tumor (black arrow heads) and associated hepatic metastatic deposits (white arrow). b Aortogram shows the tumor neovascularity predominantly from the GDA and to less extent SMA. c Zoomed GDA angiogram shows the pre-embolization neovascularity and tumoral blush. $\mathbf{d}$ Post-embolization selective GDA angiogram demonstrates stasis and reduced tumoral blush 


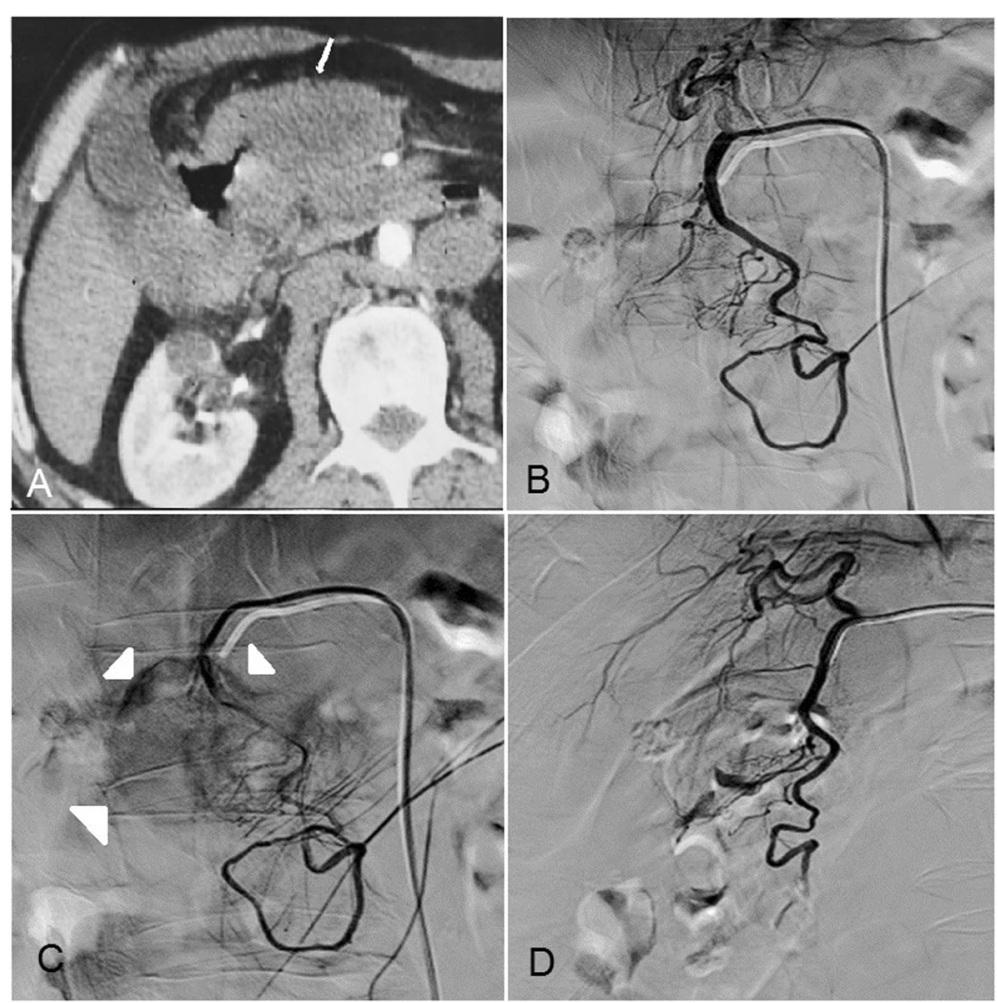

Fig. 3 a Axial CT scan for a patient with recurrent hepatic flexure mass infiltrating the duodenum (white arrow). $\mathbf{b}$ GDA angiogram shows the tumor neovascularity. c Delayed GDA angiogram shows the tumor blush (arrow heads). d Post-embolization angiograms demonstrate stasis with absent tumor blush

Table 1 Baseline demographics, presentations, tumor type, location, angiographic features, and management

\begin{tabular}{|c|c|}
\hline Age (years) Mean $\pm S D$ & mean age 55.5 years (range $42-69$ years) \\
\hline $\operatorname{Sex}(M / F)$ & $9 / 5$ \\
\hline \multicolumn{2}{|l|}{ Presentation } \\
\hline$>$ Hematemesis & $11(78.4 \%)$ \\
\hline$>$ Melena & $7(50 \%)$ \\
\hline \multicolumn{2}{|l|}{ Tumor type } \\
\hline$\triangleright$ Gastric Adenocarcinoma & $8(57.1 \%)$ \\
\hline$>$ Gastric lymphoma & $3(21.5 \%)$ \\
\hline$>\mathrm{GIST}$ & $2(14.3 \%)$ \\
\hline$>$ Colonic adenocarcinoma & $1(7.1 \%)$ \\
\hline \multicolumn{2}{|l|}{ Bleeding location } \\
\hline$>$ Gastric & $11(78.5 \%)$ \\
\hline$>$ Small Intestinal & $3(21.5 \%)$ \\
\hline \multicolumn{2}{|l|}{ Angiographic features: } \\
\hline$\triangleright$ Tumor blush & $12(85.7 \%)$ \\
\hline$>$ Neovascularity & $9(64.3 \%)$ \\
\hline$>$ Extravasation & $2(14.3 \%)$ \\
\hline \multicolumn{2}{|l|}{ Management: } \\
\hline$\triangleright$ TAE with gelatin sponge particles. & $2 / 14(14.3 \%)$ \\
\hline$>$ TAE with gelatin sponge particles and repeated procedure with PVA. & $3 / 14(21.5 \%)$ \\
\hline$>$ TAE with PVA particles. & 8/14 (57.1\%) \\
\hline
\end{tabular}




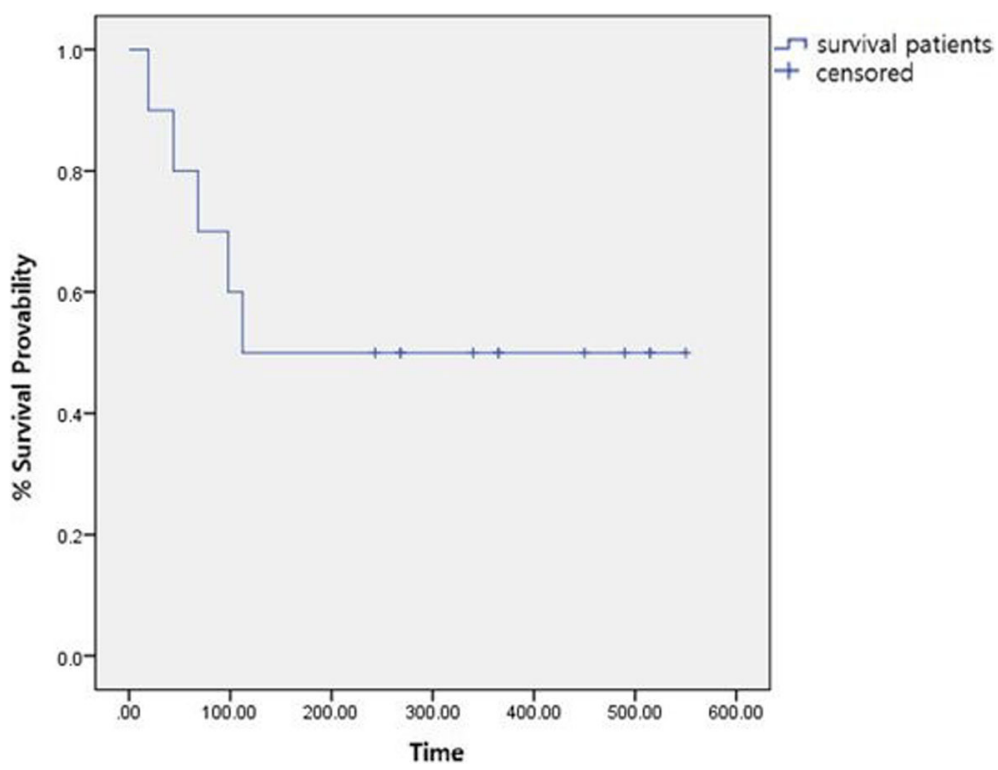

Fig. 4 Kaplan-Meier survival plot for all patients with tumor-related Gl bleeding (time represented in days)

abdominal pain, which lasted for a few days after procedures and it was controlled by oral or IV administration of non-steroidal anti-inflammatory drugs. No major procedure-related complications were identified. The average post-procedural admission period was 2 weeks.

The median post-procedural follow-up duration was 241 days. The 30 -day mortality rate was $7.1 \%(1 / 14)$ due to multi-organ failure in a patient with massive GIST metastasizing into the liver that died at day 29 after the TAE procedure. The overall mortality rate was $35.7 \%$ (5/14) which included 3 patients with unresectable gastric adenocarcinoma. The reported mortality was secondary to disease progression in 2 patients, acute massive non-controllable bleeding in 1 patient, and metastatic brain disease in 1 patient (Fig. 4). Angiographic, procedural, and post-procedural follow-up data for each patient were summarized in Table 2 (Additional file 1).

Table 2 Angiographic, procedural, post-procedural follow-up data for each patient

\begin{tabular}{|c|c|c|c|c|c|c|c|c|c|}
\hline \multirow{2}{*}{$\begin{array}{l}\text { Case } \\
\text { no. }\end{array}$} & \multirow[t]{2}{*}{ Type and site } & \multicolumn{3}{|c|}{ Angiographic findings } & \multirow[t]{2}{*}{ Endosession } & \multirow{2}{*}{$\begin{array}{l}\text { Embolic material/ } \\
\text { tools }\end{array}$} & \multirow{2}{*}{$\begin{array}{l}\text { Rebleeding } \\
\text { within } 30 \\
\text { days }\end{array}$} & \multirow{2}{*}{$\begin{array}{l}\text { Rebleeding } \\
\text { after } 30 \\
\text { days }\end{array}$} & \multirow{2}{*}{$\begin{array}{l}\text { Mortality } \\
\text { (Y/N) } \\
\text { (survival } \\
\text { days) }\end{array}$} \\
\hline & & Blush & Neovascularity & Extravasation & & & & & \\
\hline 1 & Gastric AC & Y & Y & Y & 1 & PVA & $\mathrm{N}$ & $\mathrm{N}$ & $\mathrm{Y} / 44$ \\
\hline 2 & Gastric AC & Y & $\mathrm{N}$ & $\mathrm{N}$ & 2 & Gel sponge then PVA & Y & $\mathrm{N}$ & Y/68 \\
\hline 3 & Gastric AC & $\mathrm{N}$ & Y & $\mathrm{N}$ & 1 & PVA & $\mathrm{N}$ & $\mathrm{N}$ & $\mathrm{N}$ \\
\hline 4 & Gastric AC & Y & $\mathrm{N}$ & $\mathrm{N}$ & 1 & Gel sponge & $\mathrm{N}$ & $\mathrm{N}$ & $\mathrm{N}$ \\
\hline 5 & Gastric AC & Y & Y & $\mathrm{N}$ & 1 & PVA & $\mathrm{N}$ & $\mathrm{N}$ & $\mathrm{N}$ \\
\hline 6 & Gastric AC & Y & $\mathrm{N}$ & $\mathrm{N}$ & 1 & PVA & $\mathrm{N}$ & $\mathrm{N}$ & $\mathrm{N}$ \\
\hline 7 & Gastric AC & Y & Y & Y & 1 & PVA & $\mathrm{N}$ & $\mathrm{N}$ & Y/112 \\
\hline 8 & Gastric AC & Y & Y & $\mathrm{N}$ & 1 & PVA & Y & $\mathrm{N}$ & $\mathrm{N}$ \\
\hline 9 & Gastric lymphoma & $\mathrm{N}$ & $\mathrm{N}$ & $\mathrm{N}$ & 2 & Gel sponge then PVA & $\mathrm{N}$ & Y & $\mathrm{N}$ \\
\hline 10 & Gastric lymphoma & Y & Y & $\mathrm{N}$ & 1 & PVA & $\mathrm{N}$ & $\mathrm{N}$ & $\mathrm{N}$ \\
\hline 11 & Gastric lymphoma & Y & $\mathrm{N}$ & $\mathrm{N}$ & 2 & Gel sponge then PVA & $\mathrm{N}$ & Y & $\mathrm{N}$ \\
\hline 12 & SI GIST & Y & Y & $\mathrm{N}$ & 1 & PVA & $\mathrm{N}$ & $\mathrm{N}$ & Y/29 \\
\hline 13 & SI GIST & Y & Y & $\mathrm{N}$ & 1 & PVA & $\mathrm{N}$ & $\mathrm{N}$ & $\mathrm{N}$ \\
\hline 14 & Colonic AC infiltrating Du & Y & Y & $\mathrm{N}$ & 1 & Gel sponge & Y & $\mathrm{N}$ & Y/98 \\
\hline
\end{tabular}

$A C$ adenocarcinoma, Du duodenum, $Y$ yes, $N$ no 


\section{Discussion}

In this study, we propose an alternative approach using trans-arterial embolization to manage tumor-related GI bleeding. The main finding in the current study is that technical and clinical success rates were $100 \%$ and $78.4 \%$, respectively. The clinical failure with 30 days post-procedural occurred for three patients; however, only one patient needed repeat TAE procedure.

Tumor-related GI bleeding could be a life-threatening condition that is hard to control [7]. Some patients with locally advanced or metastatic tumors are not candidates for surgical resection in addition to the limitation of endoscopic management. Reported clinical success rate for TAE as a sole management for upper GI bleeding secondary to any cause is $67 \%$ and for lower GI bleeding is $83 \%[12,13]$. To date, there are no randomized controlled trials comparing embolization to surgery for the treatment of endoscopy-refractory bleeding [14]. There are a total of six retrospective single-center case series in the published literature comparing embolization and surgery in the context of upper GI bleeding resistant to endoscopic treatment, results demonstrate no significant differences in clinical success rates or mortality between patients treated with embolization and surgery, despite the groups treated by embolization generally being older with more comorbidities [15-20].

Reports regarding the efficacy of TAE in treatment of tumoral GI bleeding are limited especially with such variability of tumor pathology and affected GI part. Nevertheless, as Tandberg et al. [21] reported in a study that included 26 patients, a $68 \%$ clinical success rate at 30 days following embolization for GI bleeding due to primary and secondary GI neoplasms. Lee et al. [22] and Meehan et al. [23] reported 52\% and $40 \%$ clinical success rates in 23 and 10 patients at 30 days following embolization performed to manage bleeding from gastric adenocarcinoma. In a more recent study by Park et al., technical and clinical success rates of TAE were $85.0 \%$ and $65.0 \%$ in 40 patients with gastric adenocarcinoma as well [24]. Additionally, Fidelman et al. [25] reported a 75\% clinical success in eight procedures done for five patients with bleeding secondary to metastatic RCC to the gastrointestinal tract treated with embolization. TAE was used to control GI bleeding secondary to lymphoma in 11 patients with reported $100 \%$ technical success rate and low clinical success rate (27\%) which was secondary to rebleeding at new sites in eight patients [26]. Furthermore, Koo et al. [27] reported 95\% and 90\% technical and clinical success rates in a study that included 20 patients in whom GI bleeding was secondary to GIST. Table 3 summarizes the reported clinical trials of tumor-related GI managed by endovascular procedures with exclusion of the case reports.
In cases of gastric tumors, the left gastric artery alone or in association with the right gastroepiploic artery was the most frequent target vessel for embolization in the current study, which was in agreement with that seen in prior reports [22-24]. The choice of target vessels for embolization should be based on the tumor sites; for tumors located in the greater curvature side, the right gastroepiploic artery could be considered and the left gastric artery could be considered for tumors located in the fundus. The gastroduodenal artery was the target vessel to embolize in cases of duodenal tumors.

The causes of TAE failure in the current study were bleeding from other newly developed tumoral growth especially in cases of gastric lymphoma (2/3). Rebleeding occurred secondary to the temporary nature of the gelatin sponge particles as embolic material (3/5) similar to Park et al., who reported rebleeding in 4 patients secondary to recanalized left gastric artery after embolization with absorbable gelatin sponge [24]. A variety of embolic materials were previously reported to control tumor-related GIT bleeding including coils, particles, and N-butyl cyanoacrylate [22-27]; however, there are no guidelines indicating the optimal choice of embolic materials. All repeat procedures in the current study were done using PVA particles to prevent recanalization of target vessels.

Complications associated with TAE may arise from non-targeted embolization and the usage of smallsized embolic particles (less than $250 \mu \mathrm{m}$ ) [26]. Ischemic complications were as splenic infarctions [24], bowel ischemia, and perforation [26]. In the current study, embolization was performed to the stomach and small bowel without any ischemic complications. Concerning mortality, previous studies reported variable mortality rates being much higher with unresectable gastric adenocarcinoma [22-27]. The main cause of mortality also in this study was unresectable adenocarcinoma $(3 / 5)$.

This study has several drawbacks owing to its nonrandomized, retrospective nature; there is heterogeneity in the patient demographics, tumor histopathology, and angiographic features. The choice of the target vessel and embolic agent varied and depended on operator preference and the use of particle embolic materials only, which could have affected the outcomes. The small number of patients precludes robust statistical analysis. Furthermore, the lack of management algorithms for such cases is related to the paucity of supporting literature.

\section{Conclusion}

Trans-arterial embolization of tumor-related gastrointestinal bleeding controlled the hemorrhage with acceptable clinical success rate and without major complications in 


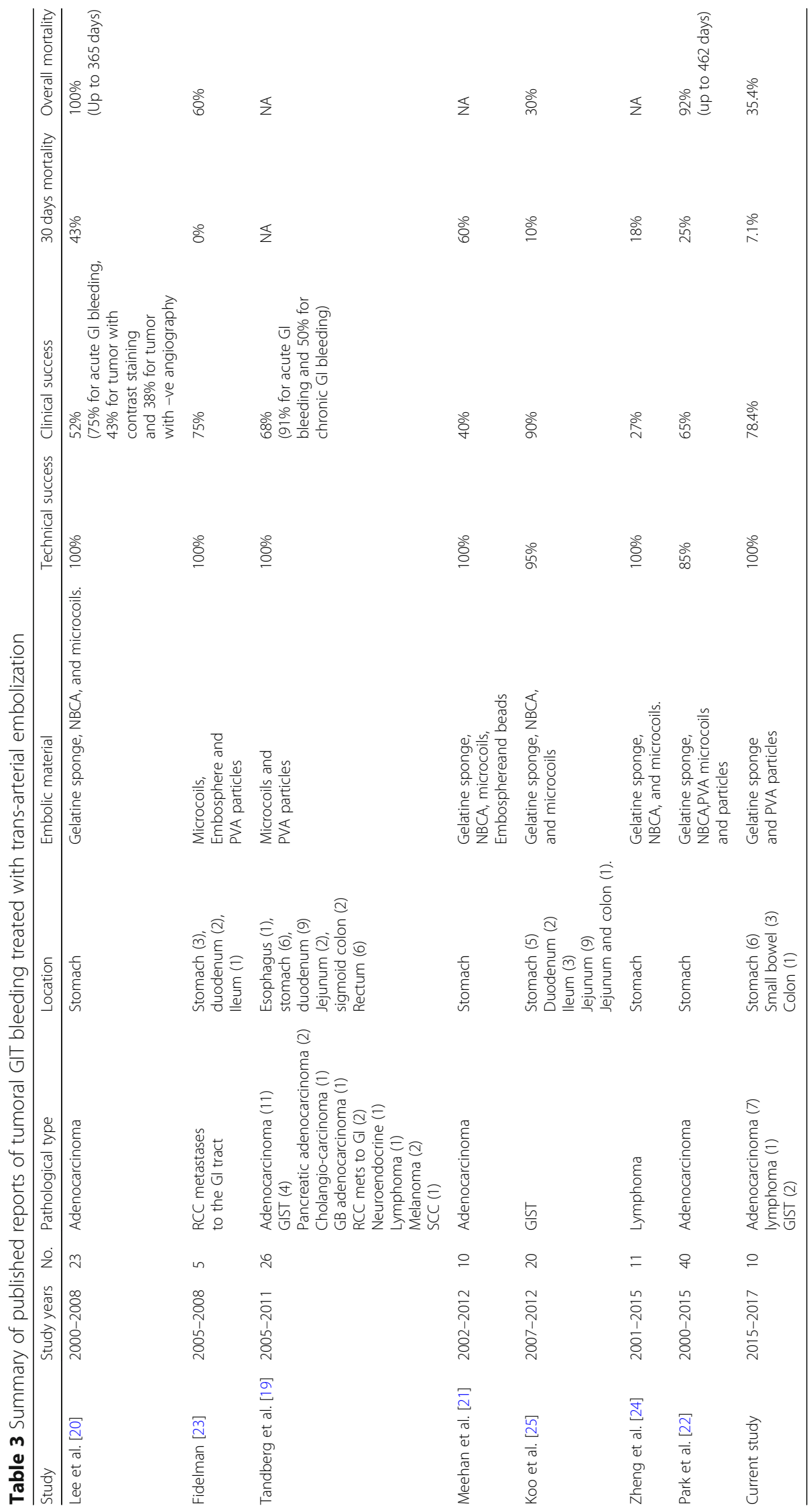


this small group of patients; therefore, it should be proposed as a symptomatic therapeutic option especially in surgically unfit patients.

\section{Supplementary information}

Supplementary information accompanies this paper at https://doi.org/10. 1186/s43055-019-0045-4.

Additional file 1. Demographics, angiographic, procedural, postprocedural follow-up data for each patient.

\section{Abbreviations}

GDA: Gastroduodenal artery; GI: Gastrointestinal; GIST: Gastrointestinal stromal tumor; PVA: Polyvinylalcohol; SMA: Superior mesenteric artery; TAE: Trans-arterial embolization

\section{Acknowledgements}

Not applicable

\section{Authors' contributions}

All authors have read and approved the manuscript. Study concept and design was proposed by AHE. Patient recruitment, follow-up, and acquisition of data were by $\mathrm{AE}$. Procedures were done by $\mathrm{AHE}, \mathrm{AH}$, and $\mathrm{AEM}$. Analysis and interpretation of data and the drafting of the manuscript were done by AHE. Revision of the manuscript was by HA and AEM. Technical or material support was by HA, AEM, and AE.

\section{Funding}

This study did not receive funding.

\section{Availability of data and materials}

All data generated or analyzed during this study are included in this published article (and its supplementary information file).

\section{Ethics approval and consent to participate}

The Mansoura University institutional review board (IRB) approved this study. All procedures performed in studies involving human participants were in accordance with the ethical standards of the institutional and/or national research committee and with the 1964 Helsinki Declaration and its later amendments or comparable ethical standards.

Informed consent was obtained from all individual participants included in the study."

\section{Consent for publication}

The participants in the study were informed and consented to the possibility of research publication. Authors hereby transfer, assign, or otherwise convey all copyright ownership to the EJRNM in the event that such work is published in that journal.

\section{Competing interests}

The authors declare that they have no competing interest.

\section{Author details}

'Diagnostic \& Interventional Radiology Department, Mansoura University, Elgomhoria St, Mansoura, Dakahlia 35516, Egypt. ${ }^{2}$ Diagnostic \& Interventional Radiology Department, Alexandria University, Alexandria, Egypt.

${ }^{3}$ Gastroenterology Surgery Center, Mansoura University, Mansoura, Egypt.

Received: 5 July 2019 Accepted: 6 September 2019

Published online: 29 October 2019

\section{References}

1. Esrailian E, Gralnek IM (2005) Non variceal upper gastrointestinal bleeding: epidemiology and diagnosis. Gastroenterol Clin North Am 34:589-605

2. Strate LL (2005) Lower Gl bleeding epidemiology and diagnosis. Gastroenterol Clin North Am 34:643-664

3. Arrayeh E, Fidelman N, Gordon RL et al (2012) Transcatheter arterial embolization for upper gastrointestinal nonvariceal hemorrhage: is empiric embolization warranted? Cardiovasc Intervent Radiol 35:1346-1354
4. Savides TJ, Jensen DM, Cohen J et al (1996) Severe upper gastrointestinal tumor bleeding: endoscopic findings, treatment, and outcome. Endoscopy 28:244-248

5. Loftus EV, Alexander GL, Ahlquist DA et al (1994) Endoscopic treatment of major bleeding from advanced gastroduodenal malignant lesions. Mayo Clin Proc 69:736-740

6. Adler DG, Leighton JA, Davila RE et al (2004) ASGE guideline: the role of endoscopy in acute non-variceal upper-Gl hemorrhage. Gastrointest Endosc 60:497-504

7. Heller SJ, Tokar JL, Nguyen MT et al (2010) Management of bleeding Gl tumors. Gastrointest Endosc 72:817-824

8. Pereira J, Phan T (2004) Management of bleeding in patients with advanced cancer. Oncologist 9:561-570

9. Blackshaw GR, Stephens MR, Lewis WG et al (2004) Prognostic significance of acute presentation with emergency complications of gastric cancer. Gastric Cancer 7:91-96

10. Kajitani T (1981) The general rules for the gastric cancer study in surgery and pathology. Jpn J Surg 11:127-139

11. Forrest JH, Finlayson N, Shearman D (1974) Endoscopy in gastrointestinal bleeding. Lancet 304:394-397

12. Mirsadraee S, Tirukonda P, Nicholson A et al (2011) Embolization for nonvariceal upper gastrointestinal tract haemorrhage: a systematic review. Clin Radiol 66:500-509

13. Darcy M (2003) Treatment of lower gastrointestinal bleeding: vasopressin infusion versus embolization. J Vasc Interv Radiol 14:535-543

14. Ripoll C, Banares R, Beceiro I et al (2004) Comparison of transcatheter arterial embolization and surgery for treatment of bleeding peptic ulcer after endoscopic treatment failure. J Vasc Interv Radiol 15:447-450

15. Eriksson LG, Ljungdahl M, Sundbom M et al (2008) Transcatheter arterial embolization versus surgery in the treatment of upper gastrointestinal bleeding after therapeutic endoscopy failure. J Vasc Interv Radiol 19:1413-1418

16. Larssen L, Moger T, Bjørnbeth BA et al (2008) Transcatheter arterial embolisation in the management of bleeding duodenal ulcers: a 5.5-year retrospective study of treatment and outcome. Scand J Gastroenterol 43: 217-222

17. Defreyne L, De Schrijver I, Decruyenaere J et al (2008) Therapeutic decision making in endoscopically unmanageable nonvariceal upper gastrointestinal hemorrhage. Cardiovasc Intervent Radiol 31:897-905

18. Venclauskas L, Bratlie SO, Zachrisson K et al (2010) Is transcatheter arterial embolization a safer alternative than surgery when endoscopic therapy fails in bleeding duodenal ulcer? Scand J Gastroenterol 45:299-304

19. Wong TC, Wong KT, Chiu PW et al (2011) A comparison of angiographic embolization with surgery after failed endoscopic hemostasis to bleeding peptic ulcers. Gastrointest Endosc 73:900-908

20. Sos TA, Lee JG, Wixson D et al (1978) Intermittent bleeding from minute to minute in acute massive gastrointestinal hemorrhage: arteriographic demonstration. AJR Am J Roentgenol 131:1015-1017

21. Tandberg DJ, Smith TP, Suhocki PV et al (2012) Early outcomes of empiric embolization of tumor-related gastrointestinal hemorrhage in patients with advanced malignancy. J Vasc Interv Radiol 23:1445-1452

22. Lee HJ, Shin JH, Yoon HK et al (2009) Transcatheter arterial embolization in gastric cancer patients with acute bleeding. Eur Radiol 19:960-965

23. Meehan T, Stecker MS, Kalva SP et al (2014) Outcomes of transcatheter arterial embolization for acute hemorrhage originating from gastric adenocarcinoma. J Vasc Interv Radiol. 25:847-851

24. Park S, Shin JH, Gwon Dl et al (2017) Transcatheter arterial embolization for gastrointestinal bleeding associated with gastric carcinoma: prognostic factors predicting successful hemostasis and survival. J Vasc Interv Radiol 28:1012-1021

25. Fidelman N, Freed RC, Nakakura EK et al (2010) Arterial embolization for the management of gastrointestinal hemorrhage from metastatic renal cell carcinoma. J Vasc Interv Radiol 21:741-744

26. Zheng L, Shin JH, Han K et al (2016) Transcatheter arterial embolization for gastrointestinal bleeding secondary to gastrointestinal lymphoma. Cardiovasc Intervent Radiol 39:1564-1572

27. Koo HJ, Shin JH, Shin S et al (2015) Efficacy and clinical outcomes of transcatheter arterial embolization for gastrointestinal bleeding from gastrointestinal stromal tumor. J Vasc Interv Radiol 26:1297-1304.e1

\section{Publisher's Note}

Springer Nature remains neutral with regard to jurisdictional claims in published maps and institutional affiliations. 